\title{
Reportação de um erro na sintaxe do instrumento WHOQOL-HIV: proposta de uma sintaxe corrigida
}

\author{
A report of an error in the WHOQOL-HIV instrument syntax: proposed \\ corrected syntax
}

\author{
Bruno Pedroso ${ }^{1,2,3}$, Luiz A. Pilatti ${ }^{1}$, Gustavo L. Gutierrez ${ }^{2}$, Celso Bilynkievycz dos \\ Santos ${ }^{1,4}$ e Claudia T. Picinin ${ }^{1,3}$ \\ 1 Universidade Tecnológica Federal do Paraná (UTFPR). Ponta Grossa, Brasil. \\ brunops3@brturbo.com.br, lapilatti@utfpr.edu.br, glgutierrez@terra.com.br, bilynkievycz@globo.com, \\ claudiapicinin_adm@yahoo.com.br \\ 2 Universidade Estadual de Campinas (UNICAMP). Campinas, Brasil \\ 3 Faculdades Integradas de Itararé (FAFIT-FACIC). Itararé, Brasil \\ 4 Universidade Estadual de Ponta Grossa (UEPG). Ponta Grossa, Brasil
}

Recebido 22 Maio 2010/Enviado para Modificação 15 Febrão 2011/Aprovado 28 Março 2011

RESUMO

O presente estudo objetiva analisar as distorções produzidas por um erro presente na sintaxe original do instrumento WHOQOL-HIV, propondo uma sintaxe com correção ao referido erro. A nova sintaxe foi testada através do software SPSS e encaminhada para pesquisadores do tema em exame para a verificação da existência de erros. Foi constado que esta atendia ao seu propósito e nenhuma irregularidade foi encontrada. Concluise que a sintaxe do WHOQOL-HIV apresenta um erro que compromete a fidedignidade deste, sendo a sintaxe proposta nesse estudo uma possibilidade de contornar tal imbróglio, ofertando maior precisão ao retratar a realidade empírica da qualidade de vida dos portadores de HIV.

Palavras-chave: Qualidade de vida, avaliação, saúde pública, Organização Mundial da Saúde, HIV (fonte: DeCS, BIREME).

\section{ABSTRACT}

This study was aimed at analysing the distortion produced by an error in the WHOQOLHIV instrument's original syntax; syntax is proposed which would correct such error. SPSS software was used for testing the new syntax which was then sent to researchers regarding the topic being considered to verify the presence of errors. It was noted that the proposed syntax met its goal and no irregularity was found. It was thus concluded that the WHOQOL-HIV syntax had an error which jeopardised this instrument's fidelity. Using the syntax proposed in this study would enable superior precision in portraying the empirical reality of the quality of life for HIV-positive people. 
Key Words: Quality of life, evaluation, public health, World Health Organization, HIV (source: MeSH, NLM).

\section{RESUMEN}

Reporte de un error en la sintaxis del instrumento WHOQOL-HIV: propuesta de una sintaxis corregida

El estudio se propuso analizar la distorsión producida por un error presente en la sintaxis original del instrumento WHOQOL-HIV, y proponer una sintaxis que corrigen tal error. La nueva sintaxis fue probada por medio del software SPSS y fue enviada a investigadores en el tema para verificar la presencia de errores. Se constató que la sintaxis propuesta logró su objetivo y no se encontraron irregularidades. Se concluyó que la sintaxis del WHOQOL-HIV presenta un error que compromete su fidelidad y que la sintaxis propuesta en este estudio permite una mayor precisión para determinar la realidad empírica de calidad de vida de los HIV positivos.

Palabras Clave: Calidad de vida, evaluación, salud pública, Organización Mundial de la Salud, HIV (fuente: DeCS, BIREME).

\footnotetext{
Síndrome da Imunodeficiência Adquirida (AIDS) é um problema de $\triangle$ saúde pública de escala global. Desde a identificação do vírus da 1 da imunodeficiência humana (HIV), os indicadores de qualidade de vida dos portadores do vírus e a mortalidade por AIDS sofreram variações acentuadas, principalmente com o advento da terapia anti-retroviral combinada.
}

O tratamento anti-retroviral combinado produziu avanços consideráveis, mesmo sem a cura, no cotidiano dos portadores do vírus. O construto multidimensional qualidade de vida, balizado pelos novos modelos que têm norteado à área da saúde, passou a exigir novas referências para análises congruentes com a realidade desse cotidiano. A avaliação de seus níveis tornou-se imperiosa.

Diversos instrumentos passaram ser utilizados para tal mensuração, considerando que a soropositividade produz uma grande variabilidade da condição clínica, principalmente entre os indivíduos assintomáticas e os sintomáticos/doentes de AIDS. O mais importante dos instrumentos de avaliação da qualidade de vida dos portadores do vírus HIV, o WHOQOL-HIV, foi desenvolvido pela Organização Mundial da Saúde (OMS).

O WHOQOL-HIV, que foi utilizado em diversos estudos publicados em importantes periódicos como AIDS Care, AIDS and Behavior, AIDS, Revista de Saúde Pública, é derivado do WHOQOL-100. O WHOQOL-100 é a versão completa do instrumento de avaliação da qualidade de vida da OMS, que possibilita a avaliação da qualidade de vida de forma global, a partir dos domínios Físico, Psicológico, Nível de Independência, Relações Sociais, Ambiente e Aspectos espirituais/Religião/Crenças pessoais. 
Os dados levantados em aplicações do WHOQOL-HIV, seguindo recomendações do Grupo WHOQOL-HIV, devem ser tabulados com o auxílio do software estatístico SPSS, utilizando uma sintaxe desenvolvida pelo Grupo WHOQOL-HIV. Após uma análise da sintaxe para o cálculo dos resultados do Grupo WHOQOL-HIV, foi constatado que há um erro presente na mesma. Tratase da inversão indevida de uma questão. $\mathrm{O}$ erro reportado altera o resultado das aplicações do WHOQOL-HIV. O objetivo do presente estudo é analisar as distorções produzidas pela sintaxe fornecida pelo Grupo WHOQOL-HIVna análise de dados resultantes do formulário do WHOQOL-HIV e, de forma adicional, propor uma sintaxe com correção ao referido erro.

\section{METODOLOGIA}

Para a realização do presente estudo, utilizou-se da análise da sintaxe do WHOQOLHIV, em que fora encontrado um erro na referida sintaxe. Constatada tal fragilidade, propôs-se uma nova sintaxe para corrigir o erro encontrado na sintaxe original do WHOQOL-HIV.

Objetivando garantir a fidedignidade da sintaxe proposta, essa fora testada a partir do software SPSS, com dados de uma aplicação do WHOQOL-HIV em um grupo de assistência a portadores do vírus HIV do Brasil, que possuía 135 pessoas cadastradas durante a realização do presente estudo. A amostra foi composta por 121 respondentes, incluindo portadores do vírus HIV assintomáticos [63], portadores do vírus HIV sintomáticos [42] e pessoas com AIDS [16]. A sintaxe corrigida foi comparada com a sintaxe original do WHOQOL-HIV.

A sintaxe proposta nesse estudo foi encaminhada para pesquisadores do tema em exame e profissionais da área de programação computacional, para que, através uma análise minuciosa, esta fosse vasculhada em busca de erros.

\section{RESULTADOS}

A partir da análise da sintaxe do WHOQOL-HIV uma inconsistência fora encontrada na sintaxe proposta pelo Grupo WHOQOL-HIV (1). No documento originalWHOQOL-HIV Instrument Users Manual (2)-publicado no idioma inglês, o referido erro também se faz presente.Sendo um instrumento amplamente utilizado em âmbito mundial, faz-se pertinente comunicar à comunidade científica a constatação de um erro na sintaxe do WHOQOL-HIV. De acordo com a sintaxe proposta pelo Grupo WHOQOL-HIV (1), a questão F2.1 apresenta escala de respostas invertida, devendo esta ser convertida. No entanto, essa conversão não deveria ocorrer. A 
questão F2.1-Você têm energia suficiente para o seu dia-a-dia?-, é apresentada no instrumento possuindo uma escala de respostas do tipo Capacidade, cujas alternativas na escala do tipo Likert variam de nada -1- a completamente -5-.

É perceptível que a alternativa 5 representa a resposta mais positiva, enquanto o valor 1 representa a resposta mais negativa. Tal equívoco fica evidenciado ao se consultar a sintaxe do instrumento WHOQOL-100 (3), onde, essa mesma questão, também denominada F2.1, não deve ser convertida. Reforçando a incidência desse erro, cabe ressaltar que a questão F2.1 é a questão que representa a faceta Energia e fadiga no instrumento WHOQOL-bref e WHOQOL-HIV-bref. Sob a denominação Q14, ambos os instrumentos não prevêem que esta deva ser convertida.

A questão F2.1 está inserida na faceta Energia e Fadiga, que, por sua vez, integra o domínio Físico. O erro descrito anteriormente ocasiona uma variação de até 25,00 pontos em uma escala centesimal na faceta Energia e fadiga. Já no domínio Físico, a variação é de 6,25 pontos.

Objetivando suprimir essa fragilidade do WHOQOL-HIV, a sintaxe original do instrumento foi corrigida, passando a assumir a configuração indicada no Quadrol .

A sintaxe corrigida segue exatamente os mesmos parâmetros da proposta pelo Grupo WHOQOL-HIV ${ }^{1}$, se diferindo desta somente pela correção da conversão indevida da questão F2.1.

Ao se comparar os efeitos do erro produzido pela questão F2.1 nos resultados do WHOQOL-HIV relativos à amostra de 121 respondentes utilizada no presente estudo, obteve-se, em uma escala centesimal, os escores de 50,85 para a faceta Dor e Desconforto e 53,90 para o domínio Físico através da sintaxe original. Já na sintaxe corrigida, os escores retornados foram de 61,91 para a para a faceta Dor e Desconforto e 56,64 para o domínio Físico.

Como pôde ser verificado, a inversão indevida da questão F2.1 ocasionou, na respectiva amostra, um desvio de $11,1 \%$ na faceta Dor e Desconforto e um desvio de 2,7 \% no domínio Físico.

Por fim, após a análise da sintaxe propostas no presente estudo, realizada por pesquisadores do tema em exame e profissionais da área de programação computacional, foi constatado que nenhuma irregularidade foi encontrada. 
Quadro 1. Procedimento para o cálculo dos escores do WHOQOL-HIV corrigido

\begin{tabular}{|c|c|}
\hline ETAPAS & SINTAXE SPSS PARA O CÁLCULOS DOS ESCORES DO WHOQOL-HIV \\
\hline $\begin{array}{l}\text { Verificar se } \\
\text { todos os } \\
100 \text { itens } \\
\text { foram } \\
\text { preenchido } \\
\text { s com } \\
\text { respostas } \\
\text { entre } 1 \text { e } 5\end{array}$ & $\begin{array}{l}\text { RECODE F11 F12 F13 F14 F21 F22 F23 F24 F31 F32 F33 F34 F501 F502 } \\
\text { F503 F504 F41 F42 F43 F44 F51 F52 F53 F54 F61 F62 F63 F64 F71 F72 F73 } \\
\text { F74 F81 F82 F83 F84 F91 F92 F93 F94 F101 F102 F103 F104 F111 F112 } \\
\text { F113 F114 F121 F122 F123 F124 F131F132 F133 F134 F141 F142 F143 F144 } \\
\text { F151 F152 F153 F154 F511 F512 F513 F514 F161 F162 F163 F164 F171 F172 } \\
\text { F173 F174 F181 F182 F183 F184 F191 F192 F193 F194 F201 F202 F203 F204 } \\
\text { F211 F212 F213 F214 F221 F222 F223 F224 F231 F232 F233 F234 F241 F242 } \\
\text { F243 F244F521 F522 F523 F524 F531 F532 F533 F534 F541 F542 F543 F544 } \\
\text { G1 G2 G3 G4 (1=1) (2=2) (3=3) (4=4) (5=5) (ELSE=SYSMIS). }\end{array}$ \\
\hline $\begin{array}{l}\text { Converter } \\
\text { as } \\
\text { questões } \\
\text { invertidas }\end{array}$ & $\begin{array}{l}\text { RECODE F11 F12 F13 F14 F22 F24 F32 F34 F72 F73 F81 F82 F83 F84 F93 } \\
\text { F94 F102 F104 F111 F112 F113 F114 F131 F154 F163 F182 F184 F222 F232 } \\
\text { F234 F501 F502 F503 F504 F514 F512 F521 F522 F523 F524 F531 F532 F533 } \\
\text { F534 F541 F542 F544 F543 (1=5) (2=4) (3=3) (4=2) (5=1). }\end{array}$ \\
\hline $\begin{array}{l}\text { Calcular os } \\
\text { escores } \\
\text { dos } \\
\text { domínios e } \\
\text { facetas }\end{array}$ & 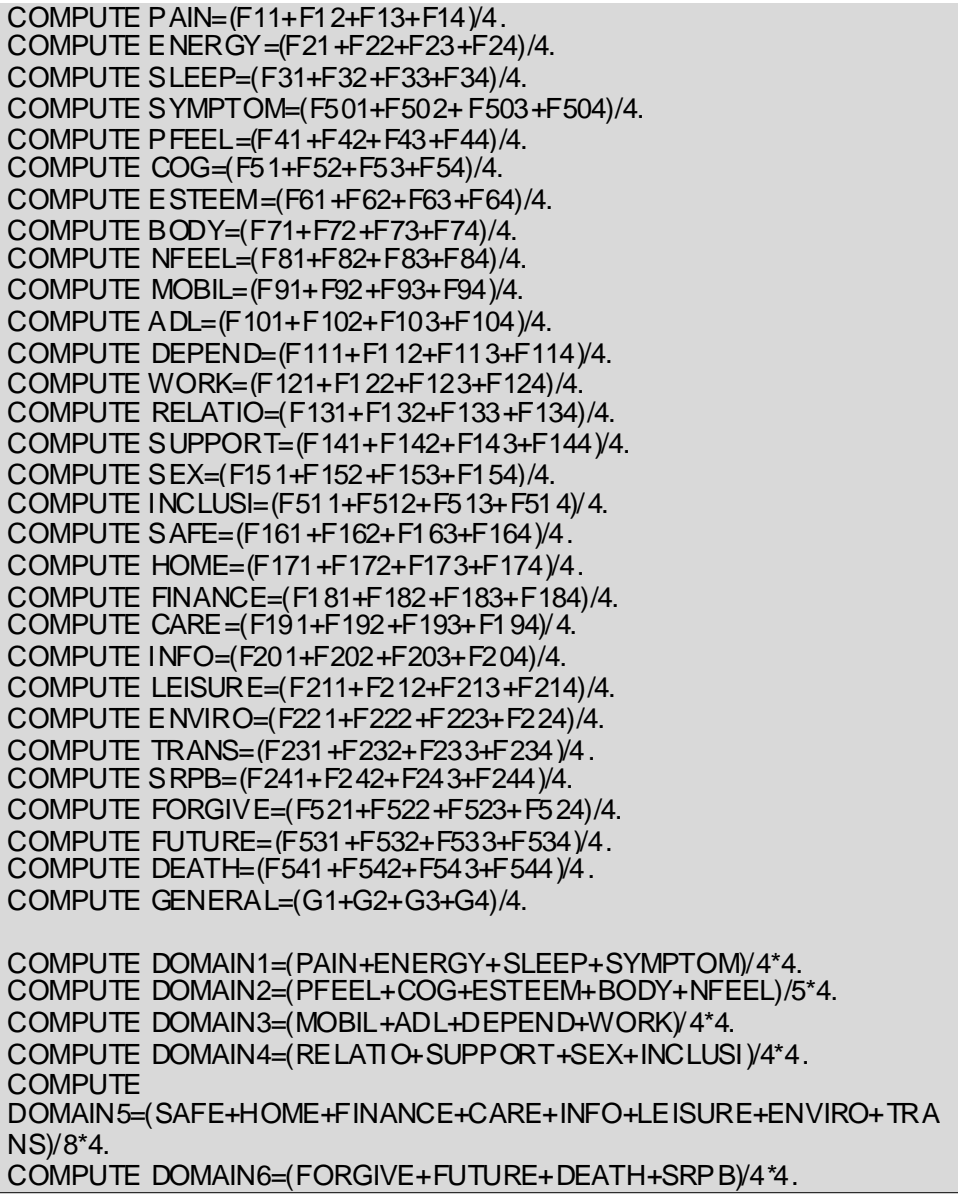 \\
\hline
\end{tabular}

Fonte: Adaptado de WHOQOL-HIV Group (2) 


\section{DISCUSSÃO}

Mesmo o WHOQOL-HIV sendo um módulo complementar do WHOQOL100, a sintaxe do instrumento não deriva por completo da sintaxe do seu precursor. Foi constatado um erro na sintaxe do WHOQOL-HIV que pode comprometer a fidedignidade do instrumento em exame: a conversão indevida da questão F2.1.

Frente à fragilidade encontrada na sintaxe do WHOQOL-HIV, fora proposta uma nova sintaxe, propondo a correção do equívoco presente na sintaxe original do WHOQOL-HIV.A sintaxe proposta neste estudo foitestada no software SPSS a partir de dados reais de pesquisa. Foi realizada uma simulação entre a sintaxe corrigida e a sintaxe original do WHOQOL-HIV. Foi solicitado para pesquisadores do tema em exame, para que a sintaxe corrigida fosse analisada e testada, objetivando a determinação de possíveis erros. Não foi retratada nenhuma incidência de erro. Constatou-se, também, que a sintaxe corrigida, factualmente, elimina o erro contido na faceta Energia e fadiga e no domínio Físico, proveniente da conversão equivocada da questão F2.1.

Conclui-se que, mesmo se tratando de um instrumento amplamente difundido, e, desenvolvido sob uma criteriosa metodologia, a sintaxe do WHOQOL-HIV apresenta um erro que pode alterar de forma significativa os resultados das aplicações do referido instrumento. Na expectativa de corrigir o erro presente na sintaxe do instrumento objeto de estudo, fora proposta uma sintaxe alternativa. Nessa perspectiva, é objetivado possibilitar uma maior fidedignidade ao WHOQOL-HIV, perfazendo com que este retrate, de forma precisa, a realidade empírica da qualidade de vida dos portadores de HIV •

\section{REFERÊNCIAS}

1. Grupo WHOQOL-HIV. Instrumento WHOQOL-HIV: sintaxe. Geneva; 2002.

2. WHOQOL-HIV Group. WHOQOL-HIV instrument users manual.Geneva; 2002.

3. WHOQOL Group. WHOQOL user manual.Geneva; 1998. 